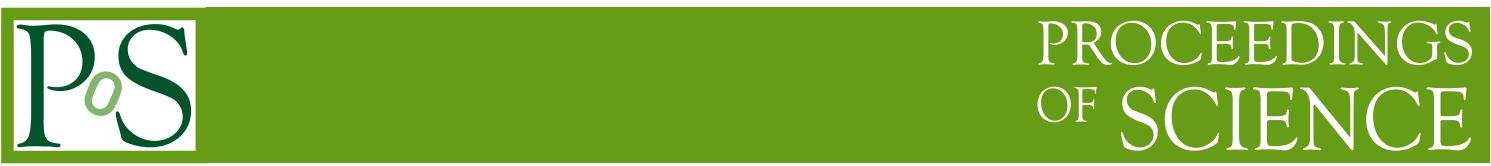

\title{
Studies of methanol maser rings
}

\author{
Anna Bartkiewicz*, Marian Szymczak \\ Torun Centre for Astronomy, Nicolaus Copernicus University, Poland \\ E-mail: [annan;msz] eastro.uni.torun.pl
}

Huib Jan van Langevelde

Joint Institute for VLBI in Europe, Dwingeloo, The Netherlands

Sterrewacht Leiden, The Netherlands

E-mail: langevelde@jive.nl

\section{James Michael De Buizer}

Stratospheric Observatory For Infrared Astronomy, USA

E-mail: jdebuizer@sofia.usra.edu

\section{Ylva Pihlström}

Department of Physics and Astronomy, University of New Mexico, USA

National Radio Astronomy Observatory, Socorro, USA

E-mail: ylva@unm.edu

\begin{abstract}
We present the results of studies of a new class of $6.7 \mathrm{GHz}$ methanol maser sources with a ringlike emission structure discovered recently with the EVN. We have used the VLA to search for water masers at $22 \mathrm{GHz}$ and radio continuum at $8.4 \mathrm{GHz}$ towards a sample of high-mass star forming regions showing a ring-like distribution of methanol maser spots. Using the Gemini telescopes we found mid-infrared (MIR) counterparts of five methanol rings with a resolution of $0 . " 15$. The centres of methanol maser rings are located within, typically, only $0 . " 2$ of the MIR emission peak, implying their physical relation with a central star. These results strongly support a scenario wherein the ring-like structures appear at the very early stage of massive star formation before either water-maser outflows or H II regions are seen.
\end{abstract}

10th European VLBI Network Symposium and EVN Users Meeting: VLBI and the new generation of radio arrays

September 20-24, 2010

Manchester, $U K$

*Speaker. 


\section{Introduction}

Methanol maser emission at $6.7 \mathrm{GHz}$ is a well known tracer of high-mass star-forming regions [5] as it lies in close surroundings of massive protostars. In 2003-07 we used the $\mathrm{EVN}^{1}$ to observe 33 methanol sources towards the Galactic plane. We imaged 31 sites and due to the increased sensitivity of EVN we detected a new morphology that had not been observed so far ([1]). In at least nine out of 31 sources the methanol maser spots showed the ring-like distribution with majoraxes from 50 to 400 mas and the ellipticies between 0.38 and 0.94 . The detailed analysis of maser spots in spectral single channel maps revealed that these structures are not consistent with rotating Keplerian discs (see Langevelde et al. this issue).

In order to find out more on the nature of the methanol masers in high-massive star-forming regions we have started three projects to investigate other signatures of high-mass formation such as $\mathrm{H}$ II regions, $\mathrm{H}_{2} \mathrm{O}$ maser emission and MIR objects. The more detailed descriptions of the results for the sample of 31 methanol masers can be found in [1], [2], while the MIR data are being prepared for publication elsewhere. Here we summarize the results obtained for nine ring-like structures.

\section{Observations and data reduction}

In order to search for radio continuum emission towards the $6.7 \mathrm{GHz}$ methanol maser rings we used the $\mathrm{VLA}^{2}$ at $8.4 \mathrm{GHz}$ in A configuration (AB1250) in the standard continuum mode on 2007 August 18. We used phase-referencing in a cycle of $50 \mathrm{~s}+250 \mathrm{~s}$ for the phase calibrator and the target, respectively, for a total of $20 \mathrm{~min}$ for each source. 3C 286 was used as a flux calibrator and two phase-calibrators were selected from a standard VLA list: J1851+0035 and J1832-1035. The data reduction was carried out following the standard recipes from AIPS Cookbook Appendix A (NRAO 2007). The final maps were created using natural weighting and the typical noise level was $\sim 50 \mu \mathrm{Jy}_{\text {beam }}{ }^{-1}\left(1 \sigma_{\mathrm{rms}}\right)$ and the beam was $0.35 \operatorname{arcsec} \times 0.25 \operatorname{arcsec}$.

We also used VLA to search for the water maser line at $22.23508 \mathrm{GHz}$ towards the methanol rings. The observations were carried on 2009 June 4 and 5 (AB1324) in CnB configuration in spectral line mode. We used a single IF 6.25-MHz wide, divided into 128 spectral channels giving a velocity resolution of $0.65 \mathrm{~km} \mathrm{~s}^{-1}$. Similarly, as in the previous project, 3C 286 was used as the primary flux density calibrator and $\mathrm{J} 1851+0035$ and $\mathrm{J} 1832-1035$ were used as phase calibrators using the same cycle time, for a total of $29 \mathrm{~min}$ for each source. The data reduction was carried out as described in the AIPS Cookbook Appendix B (NRAO 2009). The analysis was carried out on naturally weighted images, total size $77 \times 77 \operatorname{arcsec}^{2}$ and pixel size $0.15 \times 0.15 \operatorname{arcsec}^{2}$. The typical beam and the noise level per channel were $1.5 \operatorname{arcsec} \times 0.8 \operatorname{arcsec}$ and $4 \mathrm{mJy} \mathrm{beam}^{-1}$, respectively.

We used the Thermal-Region Camera and Spectrograph (T-ReCS) at Gemini South ${ }^{3}$ (GS2009B-Q-7) to image the methanol maser rings at the two MIR wavelengths $(8.7$ and $18.3 \mu \mathrm{m})$.

\footnotetext{
${ }^{1}$ The European VLBI Network is a joint facility of European, Chinese, South African and other astronomy institutes funded by their national research councils.

${ }^{2}$ The Very Large Array (VLA) of the National Radio Astronomy Observatory is a facility of the National Science Foundation operated under cooperative agreement by Associated Universities, Inc.

${ }^{3}$ Based on observations obtained at the Gemini Observatory, which is operated by the Association of Universities for Research in Astronomy, Inc., under a cooperative agreement with the NSF on behalf of the Gemini partnership:
} 
The observations were taken in several nights in the period of July-August 2009 and the total exposure time were $340 \mathrm{~s}$ at $8.7 \mu \mathrm{m}$ and $360 \mathrm{~s}$ at $18.3 \mu \mathrm{m}$. We selected five objects (G23.389+00.185 ${ }^{4}$, G23.657-00.127, G24.634-00.324, G25.411+00.105 and G26.596-00.024) for MIR observations as they showed the brightest emission at the Spitzer IRAC maps among the nine objects. We obtained images with pixel size of 0.089 arcsec and the spatial resolutions of 0.15 and 0.25 arcsec at 8.7 - and $18.3 \mu \mathrm{m}$, respectively, after deconvolution.

\section{Results and discussion}

We found that $8.4 \mathrm{GHz}$ continuum emission coincides with the methanol maser ring of G26.598 -00.024 . The separation between the centre of ellipse fitted to the methanol maser spots and the centre of the H II region is 0.8 arcsec and the maser is located to the NE from the central part of the radio continuum object (Fig. 1). Towards the other eight methanol rings we did not detect any $8.4 \mathrm{GHz}$ emission stronger than $0.15 \mathrm{mJy}$ beam $^{-1}\left(3 \sigma_{\mathrm{rms}}\right)$. That is consistent with previous findings that in general the $6.7 \mathrm{GHz}$ methanol masers do not have $\mathrm{cm}$-wavelength continuum counterparts. This implies that either the maser emission originates prior to the formation of an H II region around a massive proto-star or young star, or alternatively that the young star is too cool (i.e. too low-mass) to produce an H II region (e.g., [3]; [6]; [7]; [11]).

Searching for water maser emission resulted in a higher detection rate. Five out of nine methanol rings showed the $22 \mathrm{GHz}$ water line within $0.026 \mathrm{pc}$ (the distances are calculated for the near kinematic distances according to [8] with systemic velocities taken from [10]). We note that in 80 per cent of these objects (i.e., 4 out of 5) the position angle of the main axis of the water maser structure is crudely orthogonal $\left(60^{\circ}-120^{\circ}\right)$ to the major axis of the methanol ring, which could be interpreted as an outflow-disc scenario. However, a careful investigation of that hypothesis needs multi-epoch observations using VLBI, which we have just started using the EVN at $22 \mathrm{GHz}$. We also note that in four out of five targets the water emission is weaker than the methanol emission and that likely indicates that the outflows possibly associated with the water maser emission do not dominate these regions. The lack of water maser emission or its weakness might be related to the evolutionary stage of these regions. According to the model by [4] an outflow in massive protostar/young star starts with a collimated jet when a B-type star forms via accretion through a disk. When an $\mathrm{H}$ II region forms the wind from a massive young star produces an additional, less collimated outflow component. Later the H II region expands and a less collimated wind begins to dominate the whole system. This is an additional evidence that methanol masers are related to protostars at a very early stage of evolution - before the surrounding is ionised and before the outflow dominates the system.

Observations using Gemini enabled us to investigate MIR emission at $\sim 0.15$ arcsec resolution in five selected methanol rings. The 8- and $18 \mu \mathrm{m}$ emission appeared as extended or complex emission in four cases. A single unresolved MIR source is only found towards G23.657-00.127. We used an astrometric "triangulation" technique on the $8 \mu \mathrm{m}$ data to establish that, in four cases,

the National Science Foundation (United States), the Science and Technology Facilities Council (United Kingdom), the National Research Council (Canada), CONICYT (Chile), the Australian Research Council (Australia), Ministerio da Ciencia e Tecnologia (Brazil) and Ministerio de Ciencia, Tecnologia e Innovacion Productiva (Argentina).

${ }^{4}$ The names follow the Galactic coordinates, Gll.lll+bb.bbb. 
the offsets between the methanol ring centres and the $8-\mu \mathrm{m}$ peaks are within the $1-2 \sigma$ uncertainties only ( $0.17-0.21 \mathrm{arcsec})$ in the $8-\mu \mathrm{m}$ positions. This supports the existance of a relationship between the rings and discs or outflow cavities.

\subsection{G26.598-00.024}

We find the case of G26.598-00.024 very interesting and we will describe it in a little more detail here. It is the only methanol ring in out sample which is associated with detectable radio continuum emission. The methanol maser ring is offset by ca. $1500 \mathrm{AU}$ from the central part of the $\mathrm{H}$ II region (for the near kinematic distance of $1.85 \mathrm{kpc}$ ). The peak intensity of the radio continuum is $4.30 \mathrm{mJy}_{\text {beam }}{ }^{-1}$ and its integral flux density $42 \mathrm{mJy}$. Water maser emission appeared in two clusters that lie 1.1 (2035 AU) and $1.6 \operatorname{arcsec}(2960 \mathrm{AU})$ from the centre of the H II region to the NE, respectively. The distribution of maser spots of both species and their LSR velocities may suggest a relationship with an outflow originating from the centre of the H II region. However, the Gemini observations revealed a double structure at 8 and $18 \mu \mathrm{m}$ with a separation between two peaks of $0.97 \operatorname{arcsec}(1795 \mathrm{AU})$ at a position angle of $+45^{\circ}$ (as East of North). The NE and SW components show flux densities of 0.152 and $0.087 \mathrm{Jy}$ at $8 \mu \mathrm{m}$ and 2.24 and $0.72 \mathrm{Jy}$ at $18 \mu \mathrm{m}$. The NE component coincides within $1 \sigma$ with the centre of the methanol maser ring, while the SW MIR component coincides with the centre of the H II region. That finding makes it clear that the both masers are associated with the NE proto-/young star that has not ionised its environment yet. Interestingly, that there are no masers around the SW object which is very likely more evolved.

\section{Conclusions}

We presented a summary of three projects that studied the methanol maser emission in the massive star-forming regions. Here we focused on the results concerning the ring-like methanol masers. The maser emission appears in the very early stage of evolution of a massive proto-star or a young star, when no H II region exist and outflows are not well formed. It is possible that other morphologies would appear in later stages of evolution - when outflows form or alternatively, are created by another mechanisms such as shocks.

\section{Acknowledgements}

This work was supported by the Polish Ministry of Science and Higher Education through grant N N203 386937 and the UMK grant 378-A (2010).

\section{References}

[1] A. Bartkiewicz, M. Szymczak, H.J. van Langevelde, A.M.S. Richards \& Y.M. Pihlström The diversity of methanol maser morphologies from VLBI observations, A\&A, 2009, 502, 155

[2] A. Bartkiewicz, M. Szymczak, Y.M. Pihlström, H.J. van Langevelde, A. Brunthaler \& M.J. Reid VLA observations of water maser towards $6.7 \mathrm{GHz}$ methanol maser sources, A\&A, 2010, in press

[3] H. Beuther, A. Walsh, T.K. Sridharan, K.M. Menten \& F. Wyrowsky, $\mathrm{CH}_{3} \mathrm{OH}$ and $\mathrm{H}_{2} \mathrm{O}_{\text {masers in }}$ high-mass star-forming regions, A\&A, 2002, 390, 289-198 

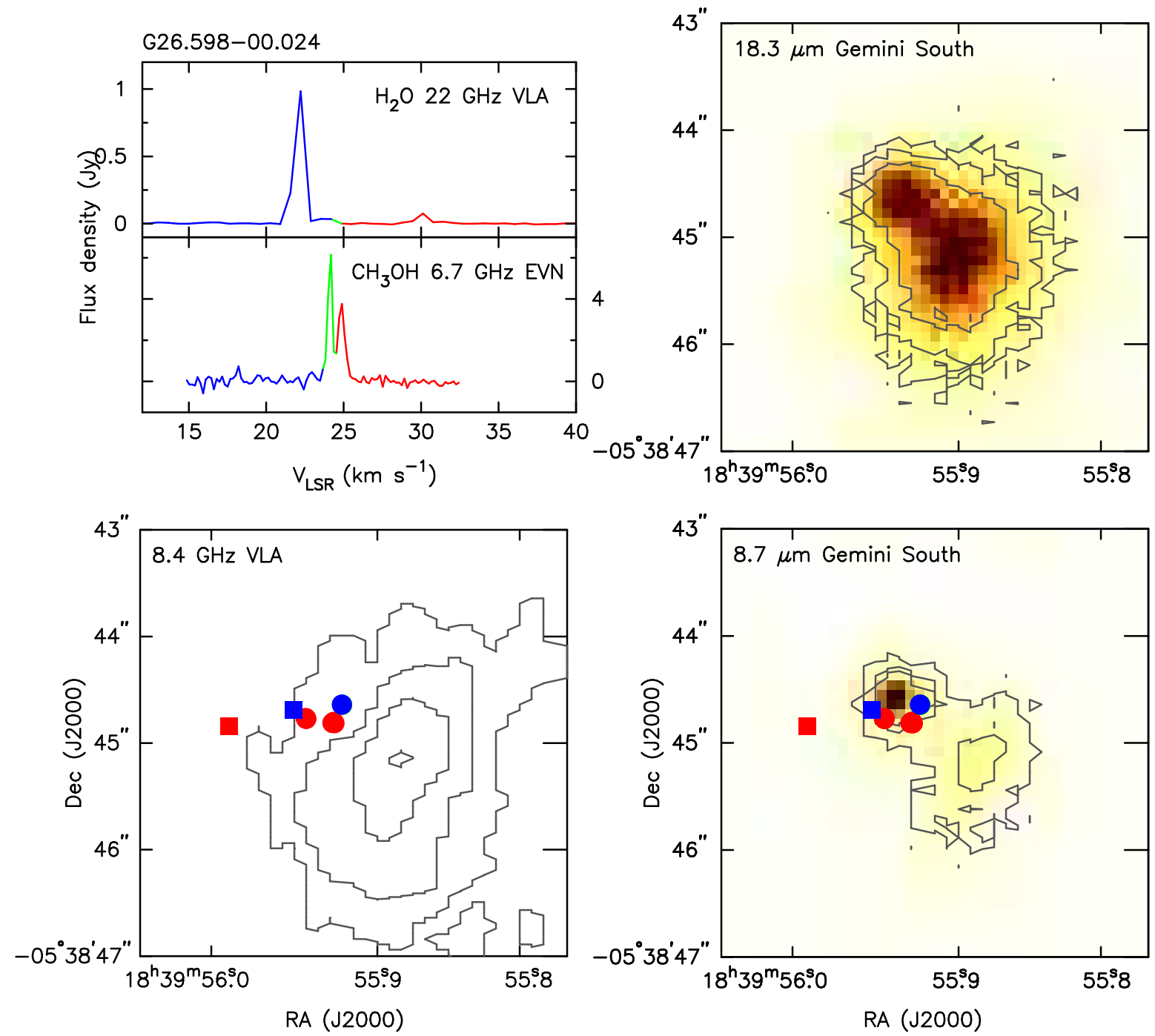

Figure 1: An overview of the G26.598-00.024 massive star-forming region. Top left: The spectra of 22 $\mathrm{GHz}$ water maser and $6.7 \mathrm{GHz}$ methanol maser lines taken using VLA and EVN, respectively. Bottom left: Distributions of methanol (circles) and water (squares) maser spots with colours corresponding to LSR velocities as indicated on the spectra. The contours trace the emission from the H II region at the levels of 3, 10 and $30 \times \sigma_{\text {rms }}$ detected using VLA at $8.4 \mathrm{GHz}$. Top and bottom right: MIR emission towards the target taken using Gemini South at 8.7 and $18.3 \mu \mathrm{m}$. The astrometry technique was used at $8.7 \mu \mathrm{m}$ with a resulting accuracy of 0.18 arcsec in RA and Dec of the registered objects.

[4] H. Beuther \& D. Shepherd, Precursors of UC H II regions and the evolution of massive outflows, arXiv:astro-ph/0502214v1, 2005

[5] K.M. Menten, The discovery of new, very strong and widespread interstellar methanol maser transition, ApJ, 1991, 380, L75-78

[6] J.D. Pandian, E. Momjian, Y. Xu, K.M. Menten \& P.F. Goldsmith, Spectral Energy Distributions of $6.7 \mathrm{GHz}$ methanol masers, A\&A, 2010, 522, 8 
[7] C.J. Phillips, R.P. Norris, S.P. Ellingsen \& P.M. McCulloch, Methanol masers and their environment at high-resolution, MNRAS, 1998, 300, 1131-1157

[8] M.J. Reid, K.M. Menten, X.W. Zheng, et al., Triginometric parallaxes of massive star-forming regions. VI. Galactic structure, fundamental parameters, and noncircular motions, ApJ, 2009, 700, 137-148

[9] M. Szymczak, A.J. Kus, G. Hrynek, A. Kepa \& E. Pazderski, 6.7 GHz methanol masers at sites of star formation. A blind survey of the Galactic plane between $20^{\circ} \leq l \leq 40^{\circ}$ and $|b| \leq 0 . .^{\circ} 52$, A\&A, 2002, 392, 277-286

[10] M. Szymczak, A. Bartkiewicz \& A.M.S. Richards, A multi-transition molecular line study of candidate massive young stellar objects associated with methanol masers, A\&A, 2007, 468, 617-625

[11] A.J. Walsh, M.G. Burton, A.R. Hyland \& G. Robinson, Studies of ultracompact H II regions, MNRAS, 1998, 301, 640-698 